\title{
Surface Roughness of Commercial Composites after Different Polishing Protocols: An Analysis with Atomic Force Microscopy
}

\author{
Luca Giacomelli ${ }^{1 *}$, Giacomo Derchi ${ }^{1}$, Andrea Frustaci ${ }^{2}$, Bruno Orlando ${ }^{1}$, Ugo Covani ${ }^{1}$, Antonio \\ Barone $^{1}$, Daniele De Santis ${ }^{2}$ and Francesco Chiappelli ${ }^{3}$ \\ ${ }^{I}$ Tirrenian Stomatologic Institute, Lido di Camaiore (Lucca), Italy \\ ${ }^{2}$ Department of Dentistry and Maxillo-facial Surgery, Faculty of Medicine, University of Verona, Italy \\ ${ }^{3}$ UCLA School of Dentistry, Division of Oral Biology and Medicine, USA
}

\begin{abstract}
:
Background and Aims: Polishing may increase the surface roughness of composites, with a possible effect on bacterial growth and material properties. This preliminary in vitro study evaluates the effect of three different polishing systems (PoGo polishers, Enhance, Venus Supra) on six direct resin composites (Gradia Direct, Venus, Venus Diamond, Enamel Plus HFO, Tetric Evoceram, Filtek Supreme XT).

Materials and Methods: For each composite, 12 square specimens were prepared: 9 specimens were polished, three for each different method, while three specimens were used as controls. Surface roughness was determined with AFM by measuring Root Mean Square (RMS) of average height.

Results: PoGo polisher determined a significantly rougher surface, versus controls, in 5 out of 6 composites evaluated. Some significant differences from unpolished controls were observed also for Enhance polishing. Polishing with Venus Supra did not result in any significant difference in surface roughness versus controls. No differences were observed between different polishing systems.

Conclusions: These preliminary results suggest that Venus Supra polishing system could determine a smoother composite surface if compared to the other polishing systems tested. On this basis, we are conducting an in vivo study to evaluate bacterial colonization on some combinations of composites and polishing protocols.
\end{abstract}

Keywords: AFM, composites, dental restoration, surface roughness.

\section{INTRODUCTION}

Survival of bacteria in the oral cavity is dependent upon adhesion to hard surfaces, such as those of teeth, filling materials, dental implants, or prostheses [1,2]. It is widely accepted that the surface roughness of intraoral hard surfaces has a major impact on the initial adhesion and the retention of oral microorganisms: in detail rougher surfaces (crowns, implant abutments, and denture bases) retain more plaque than smoother ones [1-3]. Roughness has also a major impact on the aesthetic appearance and discoloration of restorations [4], secondary caries and gingival irritation $[5,6]$ and wear of opposing and adjacent teeth [7]. In patients with less than adequate oral hygiene, variations in surface roughness of provisional restorations may be associated to the onset of subclinical or even clinical inflammation [8,9]. On the other hand, a smoother surface of intraoral structures ensures patient comfort and facilitates oral hygiene [7].

Finishing and polishing of dental restoration materials is a common clinical practice, with the aim to improve the longevity and the esthetical aspect of the composite. Dentists often provide scaling and polishing for patients at regular 6-

*Address correspondence to this author at the Tirrenian Stomatologic Institute, Via Aurelia 335, Lido di Camaiore (Lucca), Italy; Tel: +39 025693 314; Fax: +39025693 314;

E-mail: giacomelli@istitutostomatologicotirreno.it month intervals, even for those considered at low risk of developing periodontal disease. However, a recent Cochrane systematic review yielded insufficient evidence to support either the beneficial and adverse effects of routine scaling and polishing for periodontal health [10]. Moreover, an inappropriate polishing may result in a residual surface roughness, thus increasing plaque adhesion and impairing the mechanical and aesthetic characteristics of the material [11-13].

At present, several polishing protocols are used, from the "multiple-step" systems, which require different instruments, to the "one-step" systems, based on the use of unique equipment, e.g., silicon carbide brushes or rubberized cups and points permeated with diamond dust. Chromatic stability, erosion resistance and smoothness of direct restorations realized with composite resin might depend upon the material used. In fact, different composite resins present different hardness levels leading to a not uniform abrasion level after the polishing process [14]. The particle size of the composite also plays a central role: larger particles are often associated to an important detachment of the filling, and therefore to a higher porosity of the restoration [15]. Recently, the introduction of micro- and, in particular, of nano-hybrid composites has allowed to combine mechanic characteristics with a easier polishing procedure resulting, in line of principle, in a lower surface roughness [16,17]. However, experimental 
Table 1. Composites Resins Evaluated in the Present Analysis

\begin{tabular}{|c|c|c|c|}
\hline Trade name & Manufacturer & Characteristics & Granulometry \\
\hline \hline Gradia Direct & GC Corporation Tokyo & Micro-hybrid & $0.85 \mu \mathrm{m}$ \\
\hline Venus & Heraeus Kulzer srl & Micro-hybrid & $0.01-0.7 \mu \mathrm{m}$ \\
\hline Venus Diamond & Heraeus Kulzer srl & Nano-hybrid & $0.7-2 \mathrm{~nm}$ \\
\hline Enamel Plus HFO & Micerium spa & Micro-hybrid & $0.04-0.7 \mu \mathrm{m}$ \\
\hline Tetric EvoCeram & Vivadent, Schaan, Liechtenstein & Nano-hybrid & $550 \mathrm{~nm}$ \\
\hline Filtek Supreme XT & 3M ESPE, St. Paul, MN, USA & Nano-filled & $75 \mathrm{~nm}$ \\
\hline
\end{tabular}

Table 2. Polishing Systems Evaluated in the Present Analysis

\begin{tabular}{|c|c|c|}
\hline Polishing System & Manufacturer & Description \\
\hline \hline PoGo & Dentsply & diamond-impregnated resin disc \\
\hline Venus Supra & Heraeus Kulzer & silicone-impregnated polishing points \\
\hline Enhance & Dentsply & polished with aluminum oxide impregnated resin cups \\
\hline
\end{tabular}

evidence on the polishing procedure on nano-hybrid materials is still rather scant [17].

Surface roughness can be measured up to nanoscale by qualitative methods, such as scanning electron microscopy, or quantitative methods, such as profilometry [18]. In recent years, atomic force microscopy (AFM) has been largely used in dentistry to study characteristics of different materials [1921]. AFM allows a 3D imaging at a nanometric resolution, and does not need neither to work in vacuum nor any preparation of the specimen [19-21]. Of note, this technique has emerged as the most reliable in the evaluation of surface roughness [19].

On this basis, the purpose of this preliminary in vitro study is to estimate, by AFM, the surface roughness of different micro- and nano-hybrid composite resins after polishing procedures performed with different polishing systems currently in use.

\section{MATERIALS AND METHODOLOGY}

The composite resins $(n=6)$ and the polishing systems applied $(n=3)$ are summarized in Tables $\mathbf{1}$ and $\mathbf{2}$, respectively.

Square specimens $(10 \times 10 \mathrm{~mm}$, thickness $=1.5 \mathrm{~mm})$ were prepared for each resin by compressing the composite in a polyethylene matrix with an amalgam condenser. Exceeding material was removed with a cellulose stripe. The samples were then polymerized for 40 seconds with a photopolymerizing lamp.

For each composite, 12 square specimens were prepared: 9 specimens were polished, three for each different method, while three specimens were used as controls. The polishing procedure was performed always by the same trained operator according to different manufacturer's instructions, with a polishing time of 20 seconds to reproduce clinical practice. The samples obtained were kept in alcohol for 24 hours to remove detritus produced after polishing and they were kept in distilled water at $37{ }^{\circ} \mathrm{C}$ for 7 days protected from any source of light.

After one week, specimens were dried with an air jet for 30 seconds and then were observed with AFM (ELBATECH srl, Marciana, Italy), operating in tapping mode. The used probes (NSG10, NT MDT, Russia) had spring constant and resonance frequency values of approximately $10 \mathrm{~N} / \mathrm{m}$ and $250 \mathrm{kHz}$, respectively. All measurements were done in air, with $512 \times 512$ pixels surface sampling. Scan size was equal to $50 \times 50 \mu^{2}$. This area was chosen on the basis of the dimension of the typical bacteria expected to adhere to composite surface in vivo. The instrument was calibrated before the measurements using polyethylene spheres of known diameter. Five images were collected for each specimen, both in the central area and in the sides. Therefore, a total of 15 images/polishing method for each composite was collected. All AFM scans were performed by the same trained operator, who was blind towards the resin and the polishing system applied.

AFM images were analyzed using WSxM software (free downloadable from $h t t p: / / w w w . n a n o t e c . e s)$. This software was used to calculate root mean square (RMS) of the average height of every specimen, which can be assumed as a reliable index of surface roughness [19].

In this preliminary analysis, we evaluated the effects of different polishing systems on each single material, without directly comparing different composites. RMS values, expressed in $\mu \mathrm{m}$, were analyzed with descriptive statistics. Differences between polishing systems and controls were evaluated via an ANOVA test with Bonferroni's post-hoc test. A p value $<0.05$ was considered as statistically significant.

\section{RESULTS}

The average values of the surface roughness for each material analyzed with reference to the polishing protocol applied are presented in Table $\mathbf{3}$. 
Table 3. Surface Roughness in the Different Combinations of Resins/Polishing Systems Analyzed, as Derived from AFM Analysis (Scan Size: $50 \times 50 \mu \mathrm{m}^{2}$ ). All Data are Expressed as Mean RMS values \pm standard Deviations in $\mu \mathrm{m}$

\begin{tabular}{|l|c|c|c|c|c|c|}
\hline & Gradia Direct & Venus & Venus Diamond & Enamel Plus HFO & Tetric Evoceram & Filtek Supreme XT \\
& & & & & & \\
\hline PoGo polisher & $0.42 \pm 0.09^{*}$ & $0.34 \pm 0.03^{*}$ & $0.98 \pm 0.04 *$ & $0.53 \pm 0.10$ & $0.62 \pm 0.13^{*}$ & $0.78 \pm 0.13^{*}$ \\
\hline Venus Supra & $0.25 \pm 0.07$ & $0.24 \pm 0.10$ & $0.62 \pm 0.11$ & $0.45 \pm 0.02$ & $0.26 \pm 0.07$ & $0.19 \pm 0.03$ \\
\hline Enhance & $0.41 \pm 0.04$ & $0.36 \pm 0.02 *$ & $0.60 \pm 0.15$ & $0.75 \pm 0.12^{*}$ & $0.23 \pm 0.01$ & $0.16 \pm 0.02$ \\
\hline Control & $0.18 \pm 0.02$ & $0.13 \pm 0.02$ & $0.51 \pm 0.22$ & $0.40 \pm 0.05$ & $0.21 \pm 0.02$ & $0.08 \pm 0.01$ \\
\hline \multicolumn{7}{|l}{} \\
\hline${ }^{*}<0.05$ vs control
\end{tabular}

All polishing protocols resulted in a numerically higher surface roughness with respect to controls, although a statistical difference was not always observed. Overall PoGo polisher protocol resulted in slightly higher RMS values, with respect to Enhance and Venus Supra. In fact, this polishing system determined a significantly rougher surface, with respect to controls, in 5 out of 6 composites evaluated, with the exception of Enlamel Plus HFO. Some significant differences from unpolished controls were observed also for Enhance polishing, when applied to Venus and Enlamel Plus HFO. Polishing with Venus Supra did not result in any significant difference in surface roughness, with respect to controls.

However, no statistical differences were observed between different polishing systems, according to the ANOVA analysis.

\section{DISCUSSION}

The polishing phase plays a critical role in the restoration process. However, polishing may result in an increase in surface roughness, with important consequences on plaque adhesion, surface pigmentation and composite marginal integrity [11-13].

This preliminary in vitro study aimed to evaluate, by means of AFM, the surface roughness of different microand nano-hybrid composites currently used in dental restoration, after the application of different polishing protocols. In all cases, the surface roughness of polished composites was higher than unpolished controls, such suggesting that polishing determines by itself a surface damage. This finding supports the results of recent similar studies [22,23]. However, polishing of composites is often necessary to finish off the restorations with rotating devices, in order to remove any excess of material and reduce possible contacts in mouth occlusion [24].

The results of the present study, although preliminary, seem to suggest the existence of some differences in surface roughness with different polishing systems on the micro- and nanohybrid composites tested. Of note, AFM was used to evaluate the surface damage; this method has recently been proved as the most reliable method to measure surface roughness [19]. The analysis of differences between polishing systems may provide some further basis for a rationale choice of the most appropriate polishing for a given composite, among the ones tested. In most cases, Venus Supra polishing protocol resulted in a smoother surface than the other protocols tested, even if no significant differences were observed.

These differences could be likely attributed both to the intrinsic features of the composite resin, such as filling and particle size, and to the characteristics of the devices used for the polishing, from the geometry of the used tools to the hardness of the abrasive [25]. In particular, the use of "multiple-step" polishing protocols, like Venus Supra, is associated to the smoothest surface, with a roughness comparable to unpolished controls, probably because of the capability of such protocols to abrade effectively both the dispersing matrix and the particles of the filling.

It must be acknowledged that this study has several limitations, First, the in vitro nature of the present experimentation may limit, at least in part, its applicability to clinical practice. As a second limitation, although a direct correlation between surface roughness and bacterial adhesion is wellestablished [11], we did not investigate the colonization of bacteria on the polished surfaces, to seek for possible differences in the kinetic of cell growth and the hardness of bacterial plaque. Third, we did not investigate the contribution of material properties to the surface roughness resulting from polishing. Fourth, we do not have any data on the roughness of the polishing equipment, which may be correlated to the different results observed. Last, the sample size may be too small to retrieve definite conclusions; however, this analysis should be intended as a pilot study.

In fact, from the results of this pilot study, we are carrying a further investigation to evaluate, by means of AFM and fluorescence methods, the early and late colonization of bacterial cells in vivo, on same combinations of composites and polishing protocols. In this analysis, we will also evaluate material and plaque hardness.

While the results of this ongoing study will likely provide new tools for an evidence-based choice of a proper composite/polishing combination, the results of the present AFM 
analysis indicate that the composite resins tested display variable roughness depending on the polishing system used.

Overall, these preliminary results might suggest that Venus Supra polishing system could determine a smoother composite surface if compared to the other polishing systems tested.

\section{ACKNOWLEDGMENTS}

Authors are grateful to Mrs. Sara D'Imporzano for technical assistance and to Marco Salerno, PhD (Italian Institute of Technology, Genova) for useful discussion. Authors declare no conflict of interest directly relevant to this study. This study was presented at the IADR's 87 th General Session and Exhibition, Miami, 1-4 April 2009, as a poster.

\section{REFERENCES}

[1] Quirynen M, Bollen CM. The influence of surface roughness and surfacefree energy on supra-and subgingival plaque formation in man. J Clin Periodontol 1995; 22: 1-14.

[2] Sen D, Göller G, Isssever H. The effect of two polishing pastes on the surface roughness of bis-acryl composite and methacrylatebased resins. J Prosthet Dent 2002; 88: 527-32.

[3] Borchers L, Tavassol F, Tschernitschek H. Surface quality achieved by polishing and by varnishing of temporary crown and fixed partial denture resins. J Prosthetic Dent 1999; 82: 550-6.

[4] Cavalcante LM, Masouras K, Watts DC, Pimenta LA, Silikas N. Effect of nanofillers' size on surface properties after toothbrush abrasion. Am J Dent 2009; 22: 60-4.

[5] Bollen CM, Lambrechts P, Quirynen M. Comparison of surface roughness of oral hard materials to the threshold surface roughness for bacterial plaque retention: a review of the literature. Dent Mater 1997; 13: 258-69.

[6] Ono M, Nikaido T, Ikeda M, et al. Surface properties of resin composite materials relative to biofilm formation. Dent Mater J 2007; 26: 613-22.

[7] Jefferies SR. Abrasive finishing and polishing in restorative dentistry: a state-of-the-art review. Dent Clin North Am 2007; 51: 37997, ix.

[8] van Dijken JW, Sjostrom S, Wing K. Development of gingivitis around different types of composite resin. J Clin Periodontol 1987; 14: $257-60$.

[9] Janus J, Fauxpoint G, Arntz Y, Pelletier H, Etienne O. Surface roughness and morphology of three nanocomposites after two dif- ferent polishing treatments by a multitechnique approach. Dent Mater 2010; [Epub ahead of print].

[10] Beirne P, Worthington HV, Clarkson JE. Routine scale and polish for periodontal health in adults. Cochrane Database Syst Rev 2007; 4: CD004625.

[11] Kantorski KZ, Scotti R, Valandro LF, et al. Surface roughness and bacterial adherence to resin composites and ceramics. Oral Health Prev Dent 2009; 7: 29-32.

[12] Lu H, Roeder LB, Lei L, et al. Effect of surface roughness on stain resistance of dental resin composites. J Esthet Restor Dent 2005; 17: 102-8.

[13] Morgan M. Finishing and polishing of direct posterior resin restorations. Pract Proced Aesthet Dent 2004; 16: 211-7.

[14] Lutz F, Phillips RW. A classification and evaluation of composite resin systems. J Prosthet Dent 1983; 50: 480-8.

[15] Senawongse P, Pongprueksa P. Surface roughness of nanofill and nanohybrid resin composites after polishing and brushing. J Esthet Restor Dent 2007; 19: 265-73.

[16] Ritter AV. Direct resin-based composites: current recommendations for optimal clinical results. Compendium 2006; 26: 369-77.

[17] Yesil ZD, Alapati S, Johnston W, et al. Evaluation of the wear resistance of new nanocomposite resin restorative materials. J Prosthet Dent 2008; 99: 435-43.

[18] Petersilka GJ, Bell M, Häberlein I, et al. In vitro evaluation of novel low abrasive air polishing powders. J Clin Periodontol 2003; 30: 9-13.

[19] Kakaboura A, Fragouli M, Rahiotis C, et al. Evaluation of surface characteristics of dental composites using profilometry, scanning electron, atomic force microscopy and gloss-meter. J Mater Sci Mater Med 2007; 18: 155-63.

[20] Ko HC, Han JS, Bächle M, et al. Initial osteoblast-like cell response to pure titanium and zirconia/alumina ceramics. Dent Mater 2007; 23: 1349-55.

[21] Covani U, Giacomelli L, Krajewski A, et al. Biomaterials for orthopedics: a roughness analysis by atomic force microscopy. J Biomed Mater Res A 2007; 82: 723-30.

[22] Han L, Ishizaki H, Fukushima M, et al. Morphological analysis of flowable resins after long-term storage or surface polishing with a mini-brush. Dent Mater J 2009; 28: 277-84.

[23] Takanashi E, Kishikawa R, Ikeda M, et al. Influence of abrasive particle size on surface properties of flowable composites. Dent Mater J 2008; 27: 780-6.

[24] Yap AUJ, Sau CUP, Lye KW. Effects of finishing/polishing time on surface characteristics on tooth-coloured restorarives. J Oral Rehabil 1998; 25: 456-61.

[25] Stoddard J W, Johnson GH. An evaluation of polishing agents for composite resins. J Prosthet Dent 1991; 65: 491-5.

(C) Giacomelli et al.; Licensee Bentham Open.

This is an open access article licensed under the terms of the Creative Commons Attribution Non-Commercial License (http://creativecommons.org/licenses/by-nc/3.0/) which permits unrestricted, non-commercial use, distribution and reproduction in any medium, provided the work is properly cited. 